\title{
Flipped Classroom: modelo educativo centrado en el alumno
}

as tecnologías de enseñanza han cambiado dramáticamente durante los últimos 15 años. A medida que las nuevas tecnologías están disponibles, se adecuan a la innovación educativa en un intento por mejorar la enseñanza tradicional. Flipped Classroom (Aula Invertida) es uno de los modelos más recientes y populares de enseñanza, que se sustentan en el uso de la tecnología y que fue acuñado por los profesores Jonathan Bergmann y Aaron Sams del Instituto Woodland Park en Colorado, de Estados Unidos de América, en el 2007 (Bergmann y Sams, 2012). Los principios básicos del modelo de enseñanza de Aula Invertida consisten en proporcionar contenido en línea fuera del aula y motivar el aprendizaje activo en el salón, mediante actividades, para la aplicación de conceptos, facilitadas por el profesor en clase. Existen varios mecanismos para poner a disposición el contenido en línea; sin embargo, los dispositivos como los teléfonos inteligentes, las tabletas y las computadoras portátiles son elementos que se han vuelto cotidianos.

\section{Introducción}

Invertir el aula está emergiendo como un enfoque único para mejorar la retención y transferencia del estudiante, y hacer un uso eficiente del tiempo de clase (Gilboy, Heinerichs y Pazzaglia, 2015). El Aula Invertida es un modelo de aprendizaje basado en dos componentes principales, en el que el logro del contenido se traslada hacia afuera de la clase; para ello, los maestros permiten que las lecciones se encuentren disponibles para que los estudiantes puedan acceder a ellas cuando y donde les resulta conveniente, y posteriormente, en clase, aplicar los conceptos de las lecciones, estableciendo acciones facilitadas por el docente y que motiven un aprendizaje activo.

La estructura básica de las etapas de desarrollo del Aula Invertida se muestra en la figura 1. Las partes de la figura de antes de clase (pre-clase), en clase y posterior a la clase (post-clase) reflejan tres etapas generales derivadas de los principios de la teoría del aprendizaje en lugar de tecnologías específicas.

La etapa antes de clase involucra un aprovechamiento del tiempo en el aula pues, en lugar de emplearlo en informar, comunicar y crear conciencia en el grupo, el docente generalmente moverá este tipo de actividad a un entorno asíncrono fuera del aula. Por su parte, en la etapa de la clase dentro del aula, el docente propicia la interacción entre los estudiantes y con él mismo a través de debates, cuestionamientos; además, de la observación y potencialmente el uso de tecnologías, tales como los sistemas de respuesta. ${ }^{1}$ Finalmente, en la etapa posterior a la clase los docentes tienen la oportunidad de mantener e incrementar la motivación del estudiante fuera del tiempo de clase, además de evaluar el progreso del alumno.

1 Los sistemas de respuesta permiten realizar preguntas colectivas a una audiencia y recoger las respuestas individuales emitidas mediante mandos electrónicos (Bruff, 2009). 


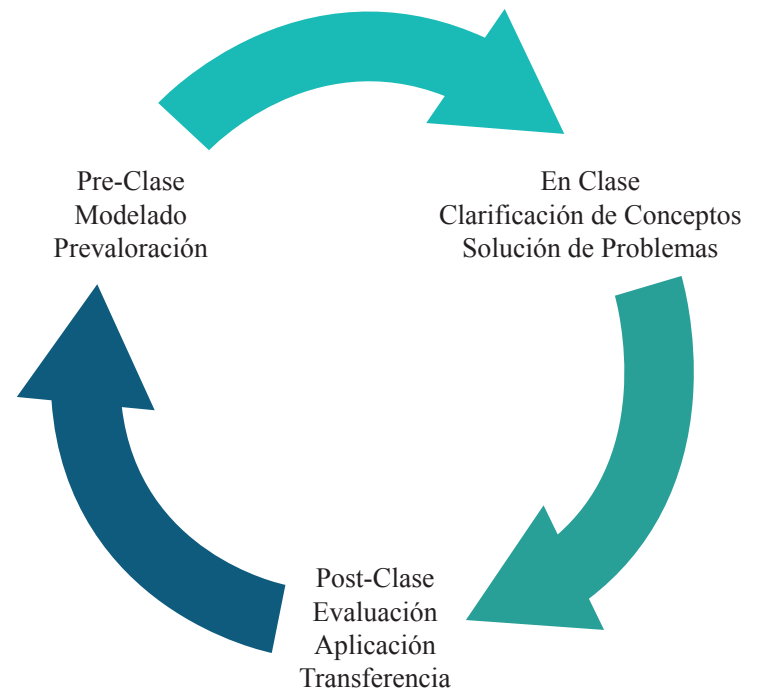

Figura 1. Etapas del Aula Invertida

Adaptada de Estes et al. (2014).

Idealmente, en cualquier curso los estudiantes interiorizarán el aprendizaje y comenzarán a transferir conocimientos y habilidades de un contexto de instrucción a otro, aplicando lo aprendido al encontrarse en situaciones reales. Existe gran variedad de métodos y herramientas disponibles para evaluar el aprendizaje de los estudiantes después de la enseñanza, tales como la rúbrica, la presentación y demostración de resultados presenciales o en línea, así como el uso de portafolios.

\section{Pilares del modelo}

De acuerdo con el modelo del ciclo de aprendizaje constructivista basado en la investigación (Bybee et al., 2006), la enseñanza consiste en dos fases: una en la que los estudiantes adquieren entendimiento conceptual, y otra más, en la que aprenden a aplicar y/o evaluar esos conceptos en situaciones novedosas, para ampliar su comprensión conceptual más allá del contexto en el que aprendieron. En el modelo de Aula Invertida los profesores desplazan el aprendizaje directo del gran espacio de aprendizaje grupal, al espacio de aprendizaje individual, con la ayuda de una de varias tecnologías (Estes et al., 2014); éstos son los elementos básicos de un Aula Invertida.
Sin embargo, al igual que con las aulas tradicionales, no hay dos aulas invertidas idénticas. Pese a que no existe un algoritmo de "cómo hacer" asociado con el modelo de Aula Invertida, hay temas unificadores. Un grupo de educadores experimentados de la Red de Aprendizaje Flipped, junto con Pearson (Network, 2014), identificaron los cuatro pilares de F-L-I-P TM, un acrónimo en inglés de Ambiente Flexible, Cultura de Aprendizaje, Contenido Intencional y Educador Profesional, estos pilares se describen a continuación:

- Ambiente flexible: este modelo presenta gran diversidad de formas de aprendizaje; en donde los docentes a menudo reorganizan físicamente su espacio educativo para adecuar la lección o unidad, pudiéndose incluir: trabajo en grupo, estudio independiente, investigación, cumplimiento y evaluación. En este tenor, los profesores aceptan que el tiempo en el aula puede llegar a ser caótico y ruidoso, en comparación con la calma típica de una clase bien comportada durante una cátedra. Además de presentar flexibilidad en las expectativas de los plazos de aprendizaje y evaluación.

- Cultura de aprendizaje: se tiene un cambio deliberado del enfoque educativo tradicional centrado en el docente a un planteamiento centrado en el estudiante, donde el tiempo asignado al aula está pensado para presentar los temas con mayor profundidad y crear oportunidades de aprendizaje más ricas. Los estudiantes pasan de ser el producto de la enseñanza al centro del aprendizaje, participando activamente en la construcción del conocimiento a través de oportunidades para intervenir y evaluar su aprendizaje de una manera significativa individualmente.

- Contenido intencional: el uso del material educativo es evaluado por los docentes para identificar: cuáles son los contenidos que deben enseñar directamente y cuáles deben permitir a los estudiantes explorar primero por su cuenta fuera del espacio de aprendizaje grupal. Además, emplean el contenido intencional para maximizar el tiempo en el salón de clases con el fin de adoptar varios métodos de instrucción, tales como estrategias 
activas de aprendizaje, trabajo colaborativo, aprendizaje basado en problemas o métodos socráticos, ${ }^{2}$ dependiendo del grado y el tema.

- Educadores profesionales: algunos críticos del Aula Invertida han sugerido que los videos instruccionales empleados en el modelo, eventualmente, reemplazarán a los educadores, lo cual es erróneo, ya que en el modelo de Aula Invertida los profesionales de la educación calificados son más importantes, debido al mayor grado de exigencia con respecto al modelo tradicional. Los educadores adecuados para trabajar con Aula Invertida son reflexivos en su práctica, interactúan entre sí para mejorar su profesión, aceptan la crítica constructiva y toleran el caos controlado en el aula.

\section{Consejos de implementación}

El aprendizaje activo es el componente central del método de enseñanza de Aula Invertida (Auster y Wylie, 2006). Los estudiantes pasan el tiempo de clase trabajando en actividades tales como resolver problemas, escribir ensayos, diseñar tareas o discutir conceptos entre ellos. Esto crea un ambiente de aprendizaje donde los alumnos aprenden interactuando con sus compañeros. Diversos estudios han demostrado que el trabajo con compañeros en clase mejora el aprendizaje general y aumenta la confianza del estudiante (Gehringer y Peddycord III, 2013). Los cuestionarios en el aula también se han utilizado como un modo efectivo de interacción entre el docente y el alumno, así como muchas nuevas tecnologías, entre ellas los clickers, ${ }^{3}$ que se han utilizado con éxito para fomentar el aprendizaje mediante pruebas (Mayer et al., 2009).

Desarrollar material para aulas invertidas es una tarea desafiante. Algunos docentes ya están grabando sus clases con el propósito de implementar cursos en línea, facilitando el uso y disponibilidad de esos recursos en sus clases invertidas

2 El método socrático es un método de dialéctica o demostración lógica para la indagación o búsqueda de nuevas ideas, conceptos o prismas subyacentes en la información (Benson, 2000).

3 Forma ordinaria de nombrar los sistemas de respuesta (Bruff, 2009).
(Toto y Nguyen, 2009). Sin embargo, esto tiende a propiciar que se tengan videos de larga duración. El material grabado no es apreciado por todos los estudiantes, ya que lo consideran demasiado largo y poco adecuado para asignaturas complejas (Gannod, Burge y Helmick, 2008). La mayoría de los estudiantes sugieren que la duración óptima de las conferencias en video debe ser de 15 a 30 minutos (Gehringer y Peddycord III, 2013).

La cantidad de tiempo requerido para preparar una clase invertida depende de la cantidad de contenido que se proporcionará a los estudiantes. Algunos docentes han informado requerir 35 horas de trabajo para crear una hora de material de clase, mientras que otros han reportado menos (Day y Foley, 2006). Los docentes también están obligados a desarrollar actividades de aprendizaje y evaluación en clase. La cantidad de tiempo necesario para calificar también aumenta a medida que las actividades semanales de aprendizaje necesitan ser evaluadas regularmente (Gannod et al., 2008).

\section{Conclusiones}

Las ventajas que presenta el modelo de Aula Invertida son muy variadas, según Bergmann, Overmyer y Wilie (2013) las tres más aplicables para una asignatura universitaria son:

1. Desarrollo de aprendizajes efectivos: los alumnos aprenden el contenido antes de ir a clases empleando recursos tecnológicos. Este tipo de adquisición de conocimiento, asociado a las habilidades en el uso de dispositivos computacionales, es una destreza que los alumnos interiorizan y les es de utilidad en una sociedad impulsada por el uso de las Tecnologías de la Información y la Comunicación (TIC), pero que no nacen con ellas.

2. Mayor compromiso en el material: durante las clases, los estudiantes realizan actividades prácticas de aprendizaje que permiten mostrar las aplicaciones, implicaciones y/o controversias asociadas al material estudiado.

3. Aumento de la interacción entre los alumnos y el profesorado: las sesiones completas en el aula se dedican a la participación e interacción entre los estudiantes, mientras 
completan las actividades de la clase. Propiciando que los docentes tengan una mayor compenetración con los estudiantes.

\section{《 En el Aula Invertida el contenido}

de enseñanza se traslada afuera

de la clase; el docente pone a disposición

de los estudiantes las lecciones

con el uso de las TIC, para su aplicación

en clase con acciones orientadas hacia el aprendizaje activo $\gg$
El aprendizaje activo es la parte medular de esta metodología y por ello es importante recalcar que más allá de las ventajas que presenta el modelo de Aula Invertida, éstas no son exclusivas, ni presentan mayores ganancias de aprendizaje o mejores actitudes que las logradas en el aula tradicional. Cuando ambos modelos utilizan un aprendizaje activo, el enfoque constructivista implica que las ganancias de aprendizaje en cualquiera de las condiciones son resultado del estilo de instrucción del aprendizaje activo, y no el orden en que el docente participa en el proceso de enseñanzaaprendizaje.
Auster, E. R., \& Wylie, K. K. (2006). Creating Active Learning in the Classroom: A Systematic Approach. Journal of Management Education, 30(2), pp. 333-353.

Benson, H. H. (2000). Socratic Wisdom The Model of Knowledge in Plato's Early Dialogues (First ed.): Oxford University Press.

Bergmann, J., Overmyer, J., \& Wilie, B. (2013). The flipped class: What it is and what it is not. The Daily Riff.

Bergmann, J., \& Sams, A. (2012). Flip Your Classroom: Reach Every Student in Every Class Every Day: Copublished by ISTE and ASCD.

Bruff, D. (2009). Classroom Response Systems ("Clickers") Retrieved 04 Enero 2017, from https://cft.vanderbilt.edu/guides-sub-pages/ clickers/\#examples

Bybee, R. W., Taylor, J. A., Gardner, A., Van Scotter, P., Powell, J. C., Westbrook, A. et al. (2006). The BSCS 5E instructional model: Origins and effectiveness. Colorado Springs, CO: BSCS, 5, pp. 88-98.

Day, J. A., \& Foley, J. D. (2006). Evaluating a web lecture intervention in a human-computer interaction course. IEEE Transactions on education, 49(4), pp. 420-431.

Estes, M., Ingram, R., \& Liu, J. C. (2014). A review of flipped classroom research, practice, and technologies. International HETL Review, 4(7).
Gannod, G. C., Burge, J. E., \& Helmick, M. T. (2008). Using the inverted classroom to teach software engineering. Paper presented at the Proceedings of the 30th international conference on Software engineering.

Gehringer, E. F., \& Peddycord III, B. W. (2013). The inverted-lecture model: a case study in computer architecture. Paper presented at the Proceeding of the 44th ACM technical symposium on Computer science education.

Gilboy, M. B., Heinerichs, S., \& Pazzaglia, G. (2015). Enhancing student engagement using the flipped classroom. Journal of nutrition education and behavior, 47(1), 109-114.

Mayer, R., Stull, A., Almeroth, K., Bimber, B., Chun, D., Bulger, M., et al. (2009). Using Technology-Based Methods to Foster Learning in Large Lecture Classes: Evidence for the Pedagogic Value of Clickers.

Network, F. L. (2014). The four pillars of FLIP ${ }^{\mathrm{TM}}$. Recuperado de http://www. flippedlearning. org/cms/lib07/VA01923112/Centricity/Domain/46/FLIP_handout_FNL_Web. pdf.

Toto, R., \& Nguyen, ${ }_{\text {H. (2009). Flipping the work }}$ design in an industrial engineering course. Paper presented at the 2009 39th IEEE Frontiers in Education Conference. 